FERMILAB-PUB-95/300-T

CPP-95-14

September 1995

DOE-ER-40757-071

hep-ph/9509308

UCD-95-29

\title{
Color-Octet Quarkonium Production at the $Z$ Pole
}

\author{
Kingman Cheung円 \\ Center for Particle Physics, University of Texas at Austin, Austin, TX 78712 \\ Wai-Yee Keung] \\ Physics Department, University of Illinois at Chicago, IL 60607-7059 \\ Fermilab, P.O. Box 500, Batavia, IL 60510 \\ Tzu Chiang Yuan[ \\ Davis Institute for High Energy Physics \\ University of California at Davis, Davis, CA 95616
}

\begin{abstract}
The direct production rate of $J / \psi$ via the color-octet mechanism is calculated at the $Z$ resonance. The color-octet production process $Z \rightarrow J / \psi q \bar{q}$ is shown to have a substantial branching ratio as well as a distinctive energy spectrum, which can be used as a powerful tool to distinguish from the color-singlet direct production of the $J / \psi$.
\end{abstract}

Despite charmonium and bottomonium have been discovered some times ago, heavy quarkonium physics has constantly drawn a lot of attentions from theorists. This is mainly due to the property of asymptotic freedom of QCD which allows theorists to calculate perturbatively the short-distance part in the production and in the decay rates of quarkonia, while the incalculable long-distance part can be factored out and absorbed into the wave-function for the ground state or its derivatives for higher orbital excitations. Confinement implies that

\footnotetext{
${ }^{1}$ Internet address: cheung@utpapa.ph.utexas .edu

${ }^{2}$ Internet address: keung@fnalv .fnal.gov

${ }^{3}$ Internet address: yuantc@ucdhep.ucdavis.edu
} 
quarkonium must be seen as a color-singlet object. The most natural assumption to make in the calculation of the short-distance part of quarkonium production or decay rate is that the heavy quark-antiquark pair inside the bound state is produced or annihilated with the same orbital angular momentum, spin, and color of the asymptotic physical quarkonium states. This simple picture dubbed as the color-singlet model [1] in the literature is derivable from QCD and has greater predictive power than other alternatives [2] like the color-evaporation model or the local duality approach.

During the past several years, a wealth of quarkonium data collected by the CDF detector at the Tevatron has posted great challenge to the naive color-singlet model. With the advance of the technology of silicon vertex detector, CDF in the 1992-1993 run was able to separate their quarkonium data into two vast classes [3]: the first class can all be traced back to a secondary displaced vertex which is a few microns away from the interaction point and presumably they are all coming indirectly from B meson decays; the second class of the data was called prompt because it comes from direct QCD production. Surprisingly, the rates for the prompt $\psi, \psi^{\prime}$, and $\chi_{c J}$ at the large transverse momentum region were observed to be orders of magnitude above the lowest order perturbative calculation based on the color-singlet model [3].

To resolve these discrepancies one needs to seek for new production mechanisms as well as new physical insights that go beyond the color-singlet model. The factorization model based on non-relativistic QCD (NRQCD) recently advocated by Bodwin, Braaten, and Lepage [4] provides a new framework to calculate the inclusive production and decay rates of quarkonium to any order in strong coupling constant $\alpha_{s}$, as well as to any order in $v^{2}$ where $v$ is the typical relative velocity of the heavy quarks inside the bound state. At the same time, the importance of fragmentation contributions to the quarkonium production at the Tevatron was also pointed out by Braaten and Yuan [5]. Parton fragmentation into quarkonium is formally of higher order in strong coupling constant but they can be enhanced over the traditional lowest order gluon-fusion mechanism [6] at sufficiently high transverse momentum. 
However, cross sections for prompt $\psi$ and $\psi^{\prime}$ production were observed to be more than an order of magnitude above the predictions based on the color-singlet model even after the inclusion of the fragmentation contributions [7, 8, 9].

One crucial feature of the factorization model is that quarkonium is not solely regarded as simply a quark-antiquark pair but rather a superposition of Fock states. In the light-cone gauge, the Fock state expansion for the $\psi$ is

$$
\begin{aligned}
|\psi\rangle & \left.=\left|c \bar{c}\left({ }^{3} S_{1}, \underline{1}\right)\right\rangle+\mathcal{O}(v)\left|c \bar{c}\left({ }^{3} P_{J}, \underline{8}\right) g\right\rangle+\mathcal{O}\left(v^{2}\right)\left(\mid c \bar{c}\left({ }^{3} S_{1}, \underline{8} \text { or } \underline{1}\right) g g\right\rangle+\left|c \bar{c}\left({ }^{1} S_{0}, \underline{8}\right) g\right\rangle\right) \\
& \left.+\mathcal{O}\left(v^{2}\right) \mid c \bar{c}\left({ }^{3} D_{J^{\prime}}, \underline{8} \text { or } \underline{1}\right) g g\right\rangle+\mathcal{O}\left(v^{3}\right)
\end{aligned}
$$

where the angular momenta of the $c \bar{c}$ pair in each Fock state is labeled by ${ }^{2 S+1} L_{J}$ with a color configuration of either $\underline{8}$ or $\underline{1}$. A novel idea proposed by Braaten and Fleming [10 to explain the CDF data on prompt $\psi\left(\psi^{\prime}\right)$ production is due to a color-octet term in the gluon fragmentation function into $\psi\left(\psi^{\prime}\right)$. This term corresponds to the case where the fragmenting gluon forms a $c \bar{c}$ pair in the color-octet ${ }^{3} S_{1}$ state at the short-distance of order $1 / m_{c}$ or smaller, and subsequently evolves non-perturbatively into the physical $\psi$ or $\psi^{\prime}$. The non-perturbative effects are represented by the NRQCD matrix element $\left\langle O_{8}^{\psi}\left({ }^{3} S_{1}\right)\right\rangle$ $\left(\left\langle O_{8}^{\psi^{\prime}}\left({ }^{3} S_{1}\right)\right\rangle\right)$ 四. These color-octet matrix elements are suppressed by order $v^{4}$ relative to the corresponding color-singlet matrix elements $\left\langle O_{1}^{\psi}\left({ }^{3} S_{1}\right)\right\rangle\left(\left\langle O_{1}^{\psi^{\prime}}\left({ }^{3} S_{1}\right)\right\rangle\right)$. On the other hand, the short-distance factor computed in perturbative theory for this color-octet process is only of order $\alpha_{s}\left(2 m_{c}\right)$, which is enhanced by a factor of $1 / \alpha_{s}^{2}$ relative to the color-singlet process. Therefore, the suppression in the color-octet matrix element can be easily compensated by the enhancement in the corresponding short-distance factor when compared with the colorsinglet process. The CDF data for $\psi\left(\psi^{\prime}\right)$ can be explained by including this color-octet term in the gluon fragmentation function and by adjusting the value for the matrix element $\left\langle O_{8}^{\psi}\left({ }^{3} S_{1}\right)\right\rangle\left(\left\langle O_{8}^{\psi^{\prime}}\left({ }^{3} S_{1}\right)\right\rangle\right)$ to fit the data [10, 11, 12].

While the color-octet mechanism can easily explain the transverse momentum spectrum of the quarkonium measured at the Tevatron, the normalization cannot be determined a 
priori. One has to rely upon the $\mathrm{CDF} \psi$ and $\psi^{\prime}$ data to extract the unknown color-octet matrix elements. In order to establish firmly our belief of the color-octet mechanism, it is therefore important to identify these color-octet quarkonium signals in other production processes. Recently, Cho and Leibovich 12 have studied color-octet quarkonium production at the Tevatron using exact tree-level calculations rather than the fragmentation approximation. This approach allows them to probe the CDF charmonium and bottomonium data at almost the whole range of transverse momentum. Braaten and Chen [13] showed that the color-octet mechanism can give rise to a distinctive signature at the upper endpoint of the energy distribution of $\psi$ at CLEO energy. In this Letter, we study the prompt quarkonium production by the color-octet mechanism at the $Z$ resonance. This work was motivated by the capability of the LEP detectors to separate the prompt $\psi$ from those coming from B decays [14]. Besides, the preliminary results of prompt $\psi$ production at LEP [14] suggested almost a factor of ten larger than the prediction of the most dominant color-singlet process via charm quark fragmentation [15, 16]. We shall show that the most important color-octet process will give rise to a branching ratio substantially larger than the most dominant colorsinglet process and is consistent with the preliminary LEP results [14]. Furthermore, the energy spectrum predicted by the color-octet production process is very soft and should be distinguishable from that produced by the most dominant color-singlet process. Hence, our consideration of $\psi$ production in $Z$ decays is crucial in verifying the importance of the color-octet mechanism in quarkonium production.

According to the factorization formalism [4], the differential rate for the inclusive production of a charmonium state $H$ with momentum $P$ from $Z$ decay can be written in a factored form:

$$
d \Gamma(Z \rightarrow H(P)+X)=\sum_{n} d \hat{\Gamma}(Z \rightarrow c \bar{c}(P, n)+X)\left\langle\mathcal{O}_{n}^{H}\right\rangle
$$

where $d \hat{\Gamma}$ is the partonic decay rate for producing a $c \bar{c}$ pair with total momentum $P$, vanishing relative momentum, and in an angular momentum and color state labeled collectively by $n$. The long-distance factor $\left\langle\mathcal{O}_{n}^{H}\right\rangle$ is the NRQCD matrix element describing the probability for 
the formation of the quarkonium state $H$ from a $c \bar{c}$ pair in the state $n$.

The leading order color-singlet processes are $Z \rightarrow \psi g g$ [17] and $Z \rightarrow \psi c \bar{c}$ [15]. Although both processes are of order $\alpha_{s}^{2}$, the latter is two orders of magnitude larger. The latter process has been interpreted as a fragmentation process in which the $c \bar{c}$ pair was first produced almost on-shell from the $Z$ decay and then followed by the fragmentation of $c$ or $\bar{c}$ into the $\psi$ [16]. Therefore, the process $Z \rightarrow \psi c \bar{c}$ is not suppressed by the quark propagator effect of order $1 / M_{Z}$ as it does in the process $Z \rightarrow \psi g g$. In the fragmentation approximation, the energy distribution of $\psi$ in the process $Z \rightarrow \psi c \bar{c}$ is given by

$$
\frac{d \Gamma}{d z}(Z \rightarrow \psi(z) c \bar{c}) \approx 2 \Gamma(Z \rightarrow c \bar{c}) \times D_{c \rightarrow \psi}(z)
$$

where

$$
D_{c \rightarrow \psi}(z)=\frac{16 \alpha_{s}^{2}\left(2 m_{c}\right)\left\langle\mathcal{O}_{1}^{\psi}\left({ }^{3} S_{1}\right)\right\rangle}{243 m_{c}^{3}} \frac{z(1-z)^{2}}{(2-z)^{6}}\left(16-32 z+72 z^{2}-32 z^{3}+5 z^{4}\right)
$$

and $z=2 E_{\psi} / M_{Z}$ with $E_{\psi}$ denotes the energy of $\psi$. Both processes involve the same matrix element $\left\langle\mathcal{O}_{1}^{\psi}\left({ }^{3} S_{1}\right)\right\rangle$ whose value can be extracted from the leptonic width to be about $0.73 \mathrm{GeV}^{3}$. Numerically, the widths for $Z \rightarrow \psi g g$ and $Z \rightarrow \psi c \bar{c}$ are about $6 \times 10^{-7} \mathrm{GeV}$ and $7 \times 10^{-5} \mathrm{GeV}$, respectively. There is also a higher order color-singlet process $Z \rightarrow q \bar{q} g^{*}$ followed by the gluon fragmentation $g^{*} \rightarrow \psi g g$ [18]. The branching ratio was estimated to be of order $10^{-6}$ only [5, 18. However, the recent results from LEP [14 showed a branching ratio of order $10^{-4}$ for prompt $\psi$ production, which is well above all the predictions from the color-singlet model.

The leading order color-octet process is of order $\alpha_{s}$ given by the process $Z \rightarrow \psi g$, for which one of the Feynman diagrams is shown in Fig. 1(a). It is understood that soft hadrons are around to match the color. The decay rate of this channel is given by

$$
\begin{aligned}
\Gamma(Z \rightarrow \psi g) & =\frac{64 \pi}{3} F g_{v}^{2} \frac{\left\langle O_{8}^{\psi}\left({ }^{1} S_{0}\right)\right\rangle}{M_{Z} M_{\psi}}(1-\xi)+\frac{16 \pi}{9} F g_{a}^{2} \frac{\left\langle O_{8}^{\psi}\left({ }^{3} S_{1}\right)\right\rangle}{M_{Z} M_{\psi}}\left(1-\xi^{2}\right) \\
& +\frac{256 \pi}{9} F g_{v}^{2} \frac{\left\langle O_{8}^{\psi}\left({ }^{3} P_{0}\right)\right\rangle}{M_{Z} M_{\psi}^{3}}\left[\frac{(1-3 \xi)^{2}}{1-\xi}+\frac{6(1+\xi)}{1-\xi}+\frac{2\left(1+3 \xi+6 \xi^{2}\right)}{1-\xi}\right]
\end{aligned}
$$


where $\xi=M_{\psi}^{2} / M_{Z}^{2}, F=\alpha \alpha_{s} /\left(x_{w}\left(1-x_{w}\right)\right), g_{v}=\frac{1}{4}-\frac{2}{3} x_{w}, g_{a}=-\frac{1}{4}$, and we have used a heavy quark spin symmetry relation $\left\langle\mathcal{O}_{8}^{\psi}\left({ }^{3} P_{J}\right)\right\rangle \approx(2 J+1)\left\langle\mathcal{O}_{8}^{\psi}\left({ }^{3} P_{0}\right)\right\rangle$. This color-octet process has a very distinctive signature that the $\psi$ has an energy equal one-half of the $Z$ mass and is recoiled by a hard gluon jet. Unfortunately, the short-distance factors are suppressed by at least one power of $1 / M_{Z}$. Numerically, the width for $Z \rightarrow \psi g$ is of order $10^{-7} \mathrm{GeV}$ which renders this process useless at the $Z$ resonance.

The dominant color-octet process actually begins at the order $\alpha_{s}^{2}$ in the process $Z \rightarrow \psi q \bar{q}$, for which one of the Feynman diagrams is shown in Fig. 1(b). Here $q$ represents $u, d, s, c$, or $b$. The energy distribution of $\psi$ for the process $Z \rightarrow \psi q \bar{q}$ is calculated, in the limit $m_{q}=0$, to be

$$
\begin{aligned}
\frac{d \Gamma}{d z}(Z \rightarrow \psi(z) q \bar{q}) & =\frac{\alpha_{s}^{2}\left(2 m_{c}\right)}{18} \Gamma(Z \rightarrow q \bar{q}) \frac{\left\langle O_{8}^{\psi}\left({ }^{3} S_{1}\right)\right\rangle}{m_{c}^{3}} \\
& \times\left[\left(\frac{(z-1)^{2}+1}{z}+2 \xi \frac{2-z}{z}+\xi^{2} \frac{2}{z}\right) \log \left(\frac{z+z_{L}}{z-z_{L}}\right)-2 z_{L}\right]
\end{aligned}
$$

where $z=2 E_{\psi} / M_{Z}, \xi=M_{\psi}^{2} / M_{Z}^{2}$, and $z_{L}=\left(z^{2}-4 \xi\right)^{1 / 2}$. The physical range of $z$ is $2 \sqrt{\xi}<z<1+\xi$. Similar expression was derived before for the process $Z \rightarrow \psi l^{+} l^{-}$in QED [19, 20]. The above exact result can be simplified into the following form in the limit $\xi \rightarrow 0$ :

$$
\frac{d \Gamma}{d z}(Z \rightarrow \psi(z) q \bar{q}) \approx \frac{\alpha_{s}^{2}\left(2 m_{c}\right)}{18} \Gamma(Z \rightarrow q \bar{q}) \frac{\left\langle O_{8}^{\psi}\left({ }^{3} S_{1}\right)\right\rangle}{m_{c}^{3}}\left[\frac{(z-1)^{2}+1}{z} \log \left(\frac{z^{2}}{\xi}\right)-2 z\right]
$$

Following the same procedures in Ref. [19], one can show that the above limit corresponds to the sum of the fragmentation processes of $Z \rightarrow q \bar{q}$ followed by $q \rightarrow \psi$ or $\bar{q} \rightarrow \psi$ and $Z \rightarrow q \bar{q} g$ followed by $g \rightarrow \psi$.

Using the value for the color-octet matrix element $\left\langle\mathcal{O}_{8}^{\psi}\left({ }^{3} S_{1}\right)\right\rangle \simeq 0.015 \mathrm{GeV}^{3}$ extracted from the fit to prompt $\psi$ data at the Tevatron [11, 12], $m_{c}=1.5 \mathrm{GeV}, M_{\psi} \approx 2 m_{c}$, and $\alpha_{s}\left(2 m_{c}\right)=0.253$, we show the energy distribution of $\psi$ for this process in Fig. 2 (solid curve). The decay width of $Z \rightarrow \psi q \bar{q}$ can also be obtained by integrating Eq. (可) over the 
physical range of $z$, and is given by

$$
\begin{aligned}
\frac{\Gamma(Z \rightarrow \psi q \bar{q})}{\Gamma(Z \rightarrow q \bar{q})} & =\frac{\alpha_{s}^{2}\left(2 m_{c}\right)}{36} \frac{\left\langle O_{8}^{\psi}\left({ }^{3} S_{1}\right)\right\rangle}{m_{c}^{3}}\left\{5\left(1-\xi^{2}\right)-2 \xi \log \xi+\left[2 \operatorname{Li}_{2}\left(\frac{\xi}{1+\xi}\right)-2 \operatorname{Li}_{2}\left(\frac{1}{1+\xi}\right)\right.\right. \\
& \left.\left.-2 \log (1+\xi) \log \xi+3 \log \xi+\log ^{2} \xi\right](1+\xi)^{2}\right\} \\
\simeq & 2.2 \times 10^{-4} .
\end{aligned}
$$

Here $\operatorname{Li}_{2}(x)=-\int_{0}^{x} \frac{d t}{t} \log (1-t)$ is the Spence function. Summing over all the quark flavors $(q=u, d, s, c, b)$, we obtain the decay width $\sum_{q} \Gamma(Z \rightarrow \psi q \bar{q}) \simeq 3.8 \times 10^{-4} \mathrm{GeV}$ and the branching ratio $\sum_{q} \operatorname{Br}(Z \rightarrow \psi q \bar{q}) \simeq 1.5 \times 10^{-4}$. Assuming the dominant prompt $\psi$ production process to be $Z \rightarrow q \bar{q} g^{*}$ with $g^{*} \rightarrow \psi+X$, in which according to color-singlet model the off-shell gluon fragments into a $\psi$ plus two perturbative gluons, DELPHI obtained the limit $\operatorname{Br}\left(Z \rightarrow q \bar{q} g^{*} ; g^{*} \rightarrow \psi+X\right)<4.1 \times 10^{-4}[14$. Since the event topology of our color-octet process is similar to this one, this limit should also be valid for our color-octet process. Therefore, our result is consistent with this data.

In Fig. 2 we compare the energy distributions of $\psi$ coming from the most important color-octet process $Z \rightarrow \psi q \bar{q}$ using Eq. (6) and the most dominant color-singlet process $Z \rightarrow \psi c \bar{c}$ using Eq. (3). For the color-octet process we have summed over all quark flavors, $q=u, d, s, c, b$, in the final state. The comparison in Fig. 2 shows a very spectacular difference between the color-octet and color-singlet processes. The spectrum for the color-octet process is very soft with a pronounced peak at the lower $z$ end, while the spectrum for the colorsinglet process is rather hard because of the nature of heavy quark fragmentation. Though the spectrum for the color-singlet process will be softened somewhat by including higher order QCD corrections [16], the spectacular difference remains unchanged. Another striking result is that the color-octet spectrum dominates over the color-singlet one for all values of z. This indicates that this color-octet contribution should not be neglected!

The energy distributions for the $\psi^{\prime}$ and $\chi_{c}$ coming from the color-octet and color-singlet processes are similar to those illustrated above for the $\psi$. The importance of the coloroctet contributions can also be shown for these two cases as well. The rates for direct $\psi^{\prime}$ 
production are roughly scaled down by a factor of 5 from those of the $\psi$ shown in Fig. 2 . Furthermore, the production of $\Upsilon$ by the color-singlet process $Z \rightarrow \Upsilon b \bar{b}$ and the color-octet process $Z \rightarrow \Upsilon q \bar{q}$ are similar to that of $\psi$, but with smaller branching ratios. Details will be presented elsewhere.

In closing, we have considered the color-octet quarkonium production at the $Z$ resonance. The dominant direct production of $\psi$ is shown to be the color-octet process $Z \rightarrow \psi q \bar{q}$, which is a few times larger than the most dominant color-singlet production process $Z \rightarrow \psi c \bar{c}$. Furthermore, the energy spectrum of the $\psi$ for the color-octet mechanism has been shown to be very different from that of the color-singlet process. Therefore, measurements of the decay width and the energy spectrum for the prompt $\psi$ production at LEP can further confirm the importance of the color-octet mechanism.

This work was supported in part by the United States Department of Energy under Grant Numbers DE-FG02-84ER40173, DE-FG03-93ER40757, and DE-FG03-91ER40674.

Note added: After this paper was submitted, similar results were reported by Cho [21].

\section{References}

[1] See, e.g., G.A. Schuler, CERN preprint, CERN-TH/95-75 (1995); CERN-TH-7170-94 (1994) and references therein.

[2] T.J. Weiler, Phys. Rev. Lett. 44, 304 (1980); V. Barger, W.Y. Keung, and R.J.N. Phillips, Phys. Lett. 91B, 253 (1980), and references therein.

[3] F. Abe et al. (CDF Collaboration), Phys. Rev. Lett. 69, 3704 (1992); Phys. Rev. Lett. 71, 2537 (1993); paper submitted to Int. Conf. on High Energy Physics, Glasgow, Scotland, July 1994, FERMILAB-CONF-94/136-E (hep-ex/9412013).

[4] G.T. Bodwin, E. Braaten, G.P. Lepage, Phys. Rev. D51, 1125 (1995). 
[5] E. Braaten and T.C. Yuan, Phys. Rev. Lett. 71, 1673 (1993); Northwestern preprint, NUHEP-TH-95-08 (hep-ph/9507398) to appear in Phys. Rev. D.

[6] E.W.N. Glover, A.D. Martin, and W.J. Stirling, Z. Phys. C38, 473 (1988).

[7] E. Braaten, M.A. Doncheski, S. Fleming, and M.L. Mangano, Phys. Lett. B333, 548 (1994).

[8] M. Cacciari and M. Greco, Phys. Rev. Lett. 73, 1586 (1994).

[9] D.P. Roy and K. Sridhar, Phys. Lett. B339, 141 (1994).

[10] E. Braaten and S. Fleming, Phys. Rev. Lett. 74, 3327(1995).

[11] M. Cacciari, M. Greco, M.L. Mangano, A. Petrelli, Phys. Lett. B356, 553 (1995).

[12] P. Cho and A.K. Leibovich, Caltech preprint, CALT-68-1988 (hep-ph/9505329); CALT68-2026 (hep-ph/9511315).

[13] E. Braaten and Y. Chen, Northwestern preprint, NUHEP-TH-95-9 (hep-ph/9508373).

[14] L3 Coll., Phys. Lett B288, 412 (1992); DELPHI Coll., Phys. Lett. B341, 109 (1994); CERN-PPE-95-145 (Oct 1995); OPAL Coll., Physics Note PN 178, submitted to EPSHEP 95 Conference, Brussels.

[15] V. Barger, K. Cheung, and W.-Y. Keung, Phys. Rev. D41, 1541 (1990).

[16] E. Braaten, K. Cheung, and T.C. Yuan, Phys. Rev. D48, 4230 (1993).

[17] W.-Y. Keung, Phys. Rev. D23, 2072 (1981).

[18] K. Hagiwara, A.D. Martin, and W.J. Stirling, Phys. Lett. B267, 527 (1991); erratumibid B316, 631 (1993).

[19] S. Fleming, Phys. Rev. D48, R1914 (1993); Phys. Rev. D50, 5808 (1994). After a typo is corrected, Eq.(3) of the second paper agrees with our Eq. (6). 
[20] L. Bergström and R.W. Robinett, Phys. Lett. B245, 249 (1990).

[21] P. Cho, Caltech preprint CALT-68-2020 (1995).

\section{Figure Captions}

1. One of the contributing Feynman diagrams for the processes (a) $Z \rightarrow \psi g$, (b) $Z \rightarrow \psi q \bar{q}$, and (c) $Z \rightarrow \psi c \bar{c}$.

2. The energy spectrum $d \Gamma / d z$ of the $\psi$ from the color-octet process $Z \rightarrow \psi q \bar{q}$ and the color-singlet process $Z \rightarrow \psi c \bar{c}$. 


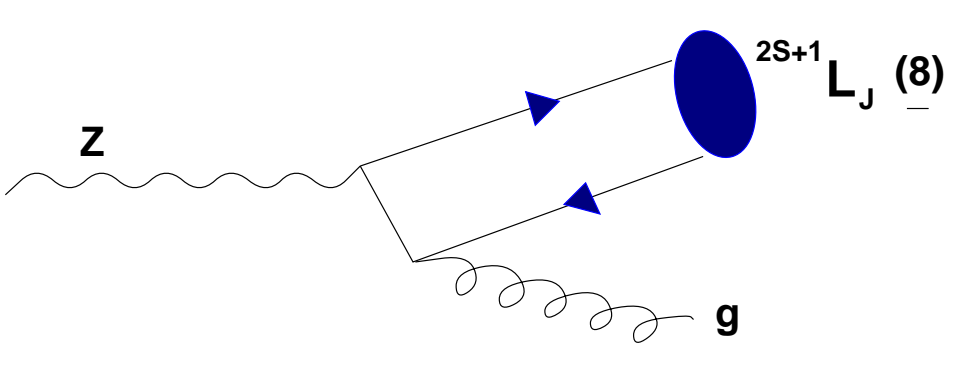

(a)

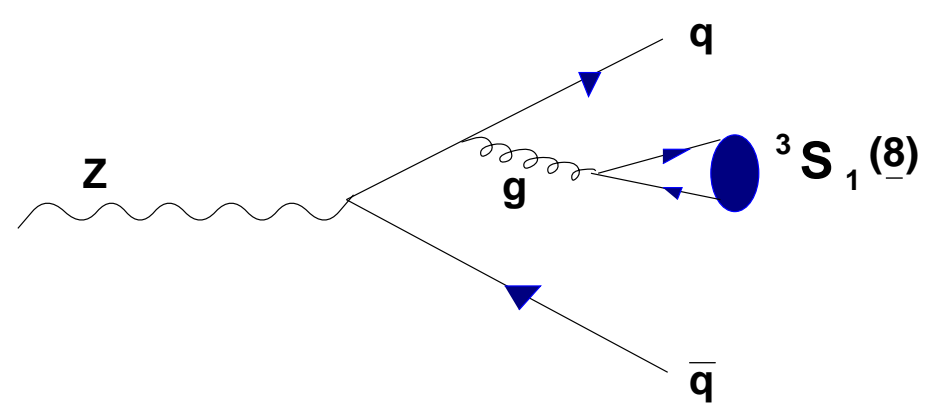

(b)

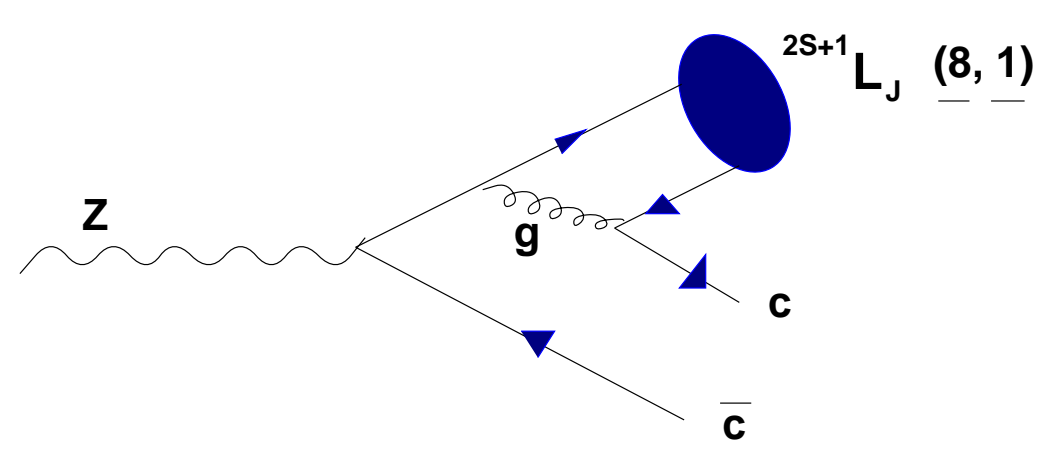

(c) 


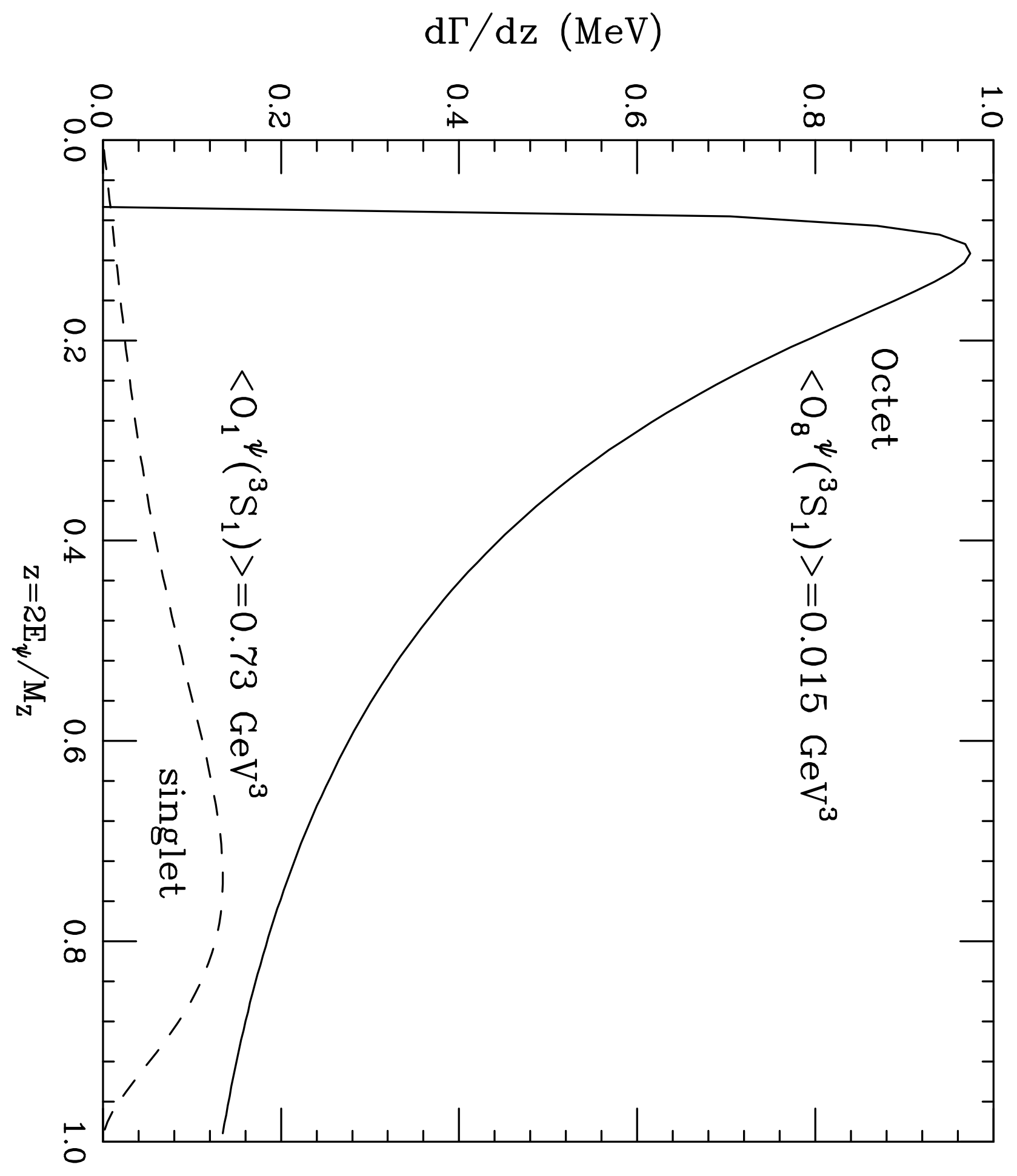

